\title{
Orthogonal test optimization Coix chinensis Tod. polysaccharide leaching process conditions
}

\author{
Yong-guang Bi , Yu-min Li \\ College of Pharmacy, Guangdong Pharmaceutical University, Guangzhou 510006, Guangdong, \\ China \\ E-mail:biyongguang2002@163.com
}

Keywords: Coix chinensis Tod. ; polysaccharides; orthogonal design; process

\begin{abstract}
Based on preliminary studies to optimize the ultrasonic extraction of polysaccharides Coix chinensis Tod. conditions using orthogonal test method, the test results showed that: In order to obtain a higher extraction rate, optimization of process parameters for each factor $A_{3} B_{3} C_{2} D_{1}$, namely ultrasonic temperature $55^{\circ} \mathrm{C}$, extraction time $50 \mathrm{~min}$, ultrasonic power $280 \mathrm{~W}$, solid-liquid ratio of $1: 10$, in this condition polysaccharide extraction rate $2.158 \%$. This method can provide a basis for future reference and process scale industrial applications.
\end{abstract}

\section{Introduction}

Polysaccharides, referred to polysaccharide is 10 or more monosaccharide groups are connected through a P bond. Are a class of proteins and nucleic acids in addition to important biological macromolecules, is one of the basic molecules of life. With the development of molecular biology, it is increasingly recognized sugar and composites have important biological activity, have been found to contain polysaccharide having a variety of traditional Chinese medicine, such as anti-tumor,immunomodulatory, hypoglycemic, anti-virus, lowering blood pressure, anti-coagulation of biological activity, such polysaccharides not cytotoxic, today has become one of the development of new drugs ${ }^{[1]}$. So far, more than 400 kinds of polysaccharide compounds from natural products are extracted, the water-soluble polysaccharide obtained from the medicine which is most important. Due to a variety of biological activity of polysaccharides and widely used in functional foods and clinical, so that the development and utilization of biological resources and research increasingly active polysaccharides become natural medicine, biochemistry, life sciences research focus.

Coix chinensis Tod.contains a variety of nutrients, and digestible and absorbed, for the elderly, women, children, Jiubingtixu, convalescence patients are good nutritious food. Zi Xu hui eyelashes addicted to the use of four oxygen selective destruction of pancreatic islet cells to establish $\mathrm{p}$ diabetic mice and found that Coix chinensis Tod. polysaccharides may inhibit glycogen breakdown, muscle glycolysis, gluconeogenesis inhibition, so as to reduce the level of blood sugar the purpose. Coix chinensis Tod. starch paste is relatively stable, retrogradation slow, difficult retrogradation, so unlike other starch products as easy to aging ${ }^{[2]}$. Unsaturated fatty acid content of Coix chinensis Tod is also higher, but also contains a strong anti-cancer activity of odd-carbon-chain fatty acids, which has alleviated the excess blood cholesterol, enhance cell membrane permeability, prevent myocardial tissue and arteriosclerosis, and other functions.

Although the high nutritional value of Coix chinensis Tod. , another medicinal value, but a Coix chinensis Tod. starch is not easy, we must be boiled after prolonged immersion when eating, which limits the use in food provoke meter area ${ }^{[3]}$. In modern process, provoke meters through the scientific process, a reasonable allocation, made of various flavors unique health care products, such as Coix chinensis Tod. wine, Coix chinensis Tod. puffed food, Coix chinensis Tod. biscuits, Coix chinensis Tod. drinks, lactic acid drinks Coix chinensis Tod. , Coix chinensis Tod. natto, easily soluble Coix chinensis Tod. milk products by consumers of all ages. I believe such a great nutritional value and health care function of the use of fresh natural products, the food industry will 
become a new economic growth point, worthy of further research and development.

Means that the frequency of electromagnetic ultrasonic waves is $20 \mathrm{kHz}$ to $50 \mathrm{MHz}$ or so, it is a mechanical wave, requires energy carrier - medium - to spread. Exists in the ultrasonic wave transmission process of alternating positive and negative pressure cycles, the positive phase, the molecules of the medium is pressed to increase the density of the original media; negative phase, the medium molecular sparse, discrete medium density decreases. That is the ultrasonic wave can not make the molecule within the sample is polarized, but the acoustic cavitation generating between solvent and sample, resulting in the formation of bubbles in the solution, the growth and blow compressed, so that dispersion of a solid sample, increasing the contact area between the sample and the extraction solvent to improve the object transfer from the solid phase to the liquid phase mass transfer rate. In industrial applications, the use of ultrasonic cleaning, drying, sterilization, and nondestructive detection of the atomization, which is a very mature technology and has wide application.

Single factor in previous work, it has been on ultrasonic extraction of polysaccharides of Coix chinensis Tod. were studied and discussed in this paper will be to optimize the ultrasonic extraction conditions using orthogonal test method, to provide a reference for future large-scale application of Coix chinensis Tod. polysaccharides.

\section{Materials and methods ${ }^{[4]}$}

\subsection{Raw materials and reagents}

Coix chinensis Tod. (Origin: Guizhou, specifications: 0.5kg/bag, Hubei Jingui Chinese Herbal Medicine Co., Ltd.). After sifted through a 40 mesh sieve to obtain honeysuckle powder and set aside. Glucose reference, Chinese medicines and biological products; phenol, concentrated sulfuric acid and deionized water, were of analytical grade.

1.2 Instruments and Equipment

H-6 constant temperature water bath (Jintan Honghua Instrument Factory); RE-52CS rotary evaporator (Gongyi City, the British valley to China Instrument Factory); I CHINABRAND circulating water pump (Gongyi City, the British valley to China instrument); UV1101 UV / Vis spectrophotometer (Shanghai Tian Mei scientific Instrument Co.); AY120 electronic analytical balance (Shimadzu Corporation).

1.3 Preparation of standard solutions of glucose

1.4 Preparation of standard curve

1.5 Determination of extraction yield of Coix chinensis Tod.

\section{Results and analysis}

The amount of Coix chinensis Tod. orthogonal to each experiment are 2.000g. In ultrasonic extraction temperature, extraction time, ultrasonic power and solid-liquid ratio of factors, each set three levels of design (see Tab.1), examine the ultrasonic extraction of polysaccharides of Coix chinensis Tod. , the results in Tab.2 and Tab.3.

As can be seen from Table 2, the four factors that affect Coix chinensis Tod. ultrasonic extraction of polysaccharides (ultrasonic time, ultrasonic temperature, solid-liquid ratio, ultrasonic power), the ultrasonic temperature maximum, minimum solid-liquid ratio on. Its impact Coix chinensis Tod. polysaccharide extraction rate of the order of magnitude has for: Ultrasound temperature $>$ ultrasonic time $>$ ultrasonic power $>$ solid-liquid ratio, ie $\mathrm{A}>\mathrm{B}>\mathrm{C}>\mathrm{D}$, on the basis of the experimental parameters, in order to obtain a higher extraction rate, each optimization of process parameters factors for $\mathrm{A}_{3} \mathrm{~B}_{3} \mathrm{C}_{2} \mathrm{D}_{1}$, namely ultrasonic temperature $55{ }^{\circ} \mathrm{C}$, extraction time $50 \mathrm{~min}$, ultrasonic power $280 \mathrm{~W}$, solid-liquid ratio of $1: 10$, in this condition polysaccharide extraction rate $2.158 \%$. 
Tab.1 Rthogonal table head design

\section{Factor}

\begin{tabular}{ccccc}
\hline Horizontal & Temperature $/{ }^{\circ} \mathrm{C}$ & Time/ min & Power $/ \mathrm{W}$ & liquid ratio /g:mL \\
& (A) & (B) & (C) & (D) \\
1 & 30 & 10 & 200 & $1: 10$ \\
2 & 45 & 40 & 280 & $1: 20$ \\
3 & 55 & 50 & 320 & $1: 30$ \\
\hline
\end{tabular}

Tab.2 Orthogonal experimental results

\begin{tabular}{cccccc}
\hline Test No. & $\mathrm{A} /{ }^{\circ} \mathrm{C}$ & $\mathrm{B} / \mathrm{min}$ & $\mathrm{C} / \mathrm{W}$ & $\mathrm{D} / \mathrm{g}: \mathrm{mL}$ & Polysaccharide extraction rate/\% \\
\hline 1 & $1(30)$ & $1(10)$ & $1(200)$ & $1(1: 10)$ & 1.543 \\
2 & 1 & $2(40)$ & $2(280)$ & $2(1: 20)$ & 1.766 \\
3 & 1 & $3(50)$ & $3(320)$ & $3(1: 30)$ & 1.667 \\
4 & $2(45)$ & 1 & 3 & 2 & 1.602 \\
5 & 2 & 2 & 1 & 3 & 1.769 \\
6 & 2 & 3 & 2 & 1 & 1.825 \\
7 & $3(55)$ & 1 & 2 & 3 & 1.734 \\
8 & 3 & 2 & 3 & 1 & \\
9 & 3 & 3 & 1 & 2.149 \\
$\mathrm{~K} 1$ & 4.977 & 4.878 & 5.250 & 5.460 & \\
$\mathrm{~K} 2$ & 5.196 & 5.415 & 5.517 & 5.325 & \\
$\mathrm{~K} 3$ & 5.763 & 5.640 & 5.196 & 5.151 & \\
$\mathrm{k} 1$ & 1.659 & 1.626 & 1.750 & 1.820 & \\
$\mathrm{k} 2$ & 1.732 & 1.805 & 1.839 & 1.775 & \\
$\mathrm{k} 3$ & 1.921 & 1.880 & 1.723 & 1.717 & \\
$\mathrm{R}$ & 0.262 & 0.254 & 0.116 & 0.103 & \\
\hline
\end{tabular}

\section{Conclusions}

On the basis of preliminary studies on the optimization of the ultrasonic extraction of polysaccharides Coix chinensis Tod. conditions using orthogonal test method, the test results showed that: In order to obtain a higher extraction rate, optimization of process parameters for each factor $\mathrm{A}_{3} \mathrm{~B}_{3} \mathrm{C}_{2} \mathrm{D}_{1}$, namely ultrasonic temperature $55^{\circ} \mathrm{C}$, ultrasonic time $50 \mathrm{~min}$, ultrasonic power $280 \mathrm{~W}$, solid-liquid ratio of $1: 10$, in this condition polysaccharide extraction rate $2.158 \%$. 
Tab.3 Orthogonal analysis of variance table

\begin{tabular}{cccccc}
\hline Factor & $\begin{array}{c}\text { Sum of squared } \\
\text { deviations }\end{array}$ & $\begin{array}{c}\text { Degrees of } \\
\text { freedom }\end{array}$ & F ratio & $\begin{array}{c}\text { F critical } \\
\text { value }\end{array}$ & Significant \\
\hline Temperature & 0.110 & 2 & 6.875 & 19.000 & \\
Time & 0.102 & 2 & 6.375 & 19.000 & \\
Power & 0.022 & 2 & 1.375 & 19.000 & \\
Liquid ratio & 0.016 & 2 & 1.000 & 19.000 & \\
Error & 0.02 & 2 & & & \\
\hline
\end{tabular}

\section{Acknowledgements}

This work was financially Supported by the Dean Project of Guangxi Key Laboratory of Petrochemical Resource Processing and Process Intensification Technology（2012K09）

\section{References}

[1]Peat S., Trkey J., ReesD.A. Carbohydratesoftheredalga,PorPhyra umbilicalis[J]. Chem.Soe., 1961,(19):1590-1595.

[2]ReesD.A.Estimating of the relative amounts of isomeric sulPhate esters in some sulPhated Polysaceharides[J].Chem.Soe.,1961,(32):5168-5171.

[3]Rees D.A,Conway E..The structure and biosythesis of Porphyran:a comparison of some samples[J].Bioehem.,1962,(84):411-416.

[4]Yong-guang Bi, Yu-min Li. Study on the ultrasonic extraction of polysaccharides of barley rice[J]. Applied Mechanics and Materials, 2014, (513-517):332-335. 